\title{
A Model for Variations of Sound Speed and Attenuation from Seabed Gas Emissions
}

\author{
Jianghui Li, Paul R. White, Jonathan M. Bull ${ }^{\dagger}$, Timothy G. Leighton, Ben Roche ${ }^{\dagger}$ \\ Institute of Sound and Vibration Research, University of Southampton SO17 1BJ, U.K. \\ ${ }^{\dagger}$ Ocean and Earth Science, University of Southampton, National Oceanography Centre, Southampton SO14 3ZH, U.K. \\ E-mail: J.Li@ soton.ac.uk
}

\begin{abstract}
The release of greenhouse gas, such as carbon dioxide $\left(\mathrm{CO}_{2}\right)$ and methane $\left(\mathrm{CH}_{4}\right)$ into the atmosphere, is a major source of global warming. As such a large emphasis has been placed on developing methods of measuring the amount of gas escaping from natural seep sites, particularly in the marine environment. Fortunately, gaseous bubbles crossing the seabed interface into the water column are relatively easily detected and quantified by passive acoustics. Here we design an active acoustic model to determine the frequency dependent changes in sound speed and attenuation in the water column due to gaseous $\mathrm{CO}_{2}$. Our approach is to formulate a numerical model simulating the propagation of sound energy at different frequencies through a mixed media incorporating a gas bubble plume with detection on a hydrophone.
\end{abstract}

Index Terms-Sound speed, attenuation, gas leakage, active acoustics, greenhouse gas, model

\section{INTRODUCTION}

In recent years, intensive research efforts have been launched by acousticians and oceanographers geared towards measurements of seabed gas emissions, both in controlled release (e.g., carbon dioxide $\left.\left(\mathrm{CO}_{2}\right)\right)$ [1]-[9] and natural seeps (e.g., submarine methane $\left.\left(\mathrm{CH}_{4}\right)\right)$ [10]-[13]. While these measurements are relatively easily conducted via optical and passive acoustic methods [9], active acoustic techniques are more complicated, with information on the variations of sound speed and attenuation resulted from the seabed gas emissions is still scarce.

The presence of gas emissions can be modelled as discrete gas flares emerging the seabed, which introduce variations in sound speed and attenuation as sound energy propagates the water column. Such variations affect signal transmission and detection in underwater acoustics [14]-[22]. As for the acoustic implications of the presence of bubbles, only a few direct measurements of the sound speed in the gas plume have been reported. King [23] measured sound-speed and attenuation at different frequencies in the presence of bubble plume from a tank bed in the laboratory. These measurements have essentially confirmed the expected perturbation of sound speed and attenuation and void fraction measurements over gas emissions underwater. A comprehensive generation of gas plume was set forth by Leifer [24] who, based on ocean measurements and previous parameterizations [25], proposed a model of formation of seep bubble plumes. Clay [26] derived a model of compressional speed of sound and attenuation in a bubble medium, from the effective density and the effective compressibility of the bubble medium. This model provides a breakthrough point to estimate the sound speed and attenuation resulted from the seabed gas emissions.

In this paper, we develop a numerical model, as shown in Fig. 1, to simulate the propagation of sound energy through a mixed media involving a gas bubble plume and detection at a hydrophone. This model parameterizes gas plumes of the assemblage of bubbles out of the seabed in sound speed and attenuation. Their variations are calculated from gas parameters, ambient parameters, flow rate, bubble size distribution, and the plume size. The numerical model is used to determine the frequency dependent changes in sound speed and attenuation in the water column due to gaseous $\mathrm{CO}_{2}$.

This paper is organized as follows. Section II introduces the formations of gas plumes emitted from seabed, while Section III describes the acoustic method on calculations of the sound speed and attenuation in the presence of gas plumes. Section IV displays the simulation results with the proposed model, taking $\mathrm{CO}_{2}$ and $\mathrm{CH}_{4}$ gas plumes as two examples. Section V describes the statistical fluctuation of the sound speed and attenuation, and Section VI completes the paper with concluding remarks and discussions.

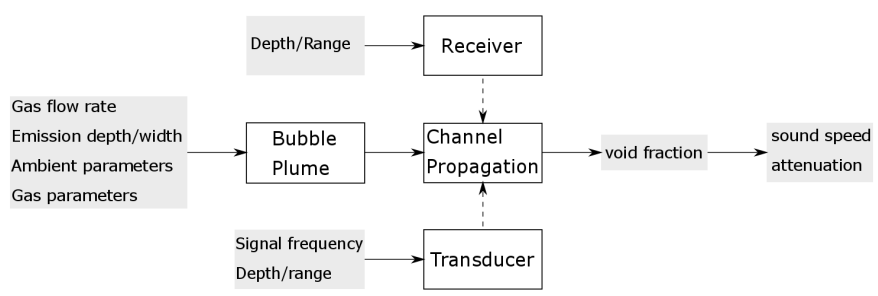

Fig. 1: Block diagram of the model. The input parameters and output data are shown in the grey blocks.

\section{FORMATION OF BUBBLE PLUME}

We employ the mechanism proposed by Leifer [24] as the basic plume framework for gas transport from seabed to surface. The gas plume is modeled as bubbles generated at the seafloor, moving randomly in horizontal direction and accelerating in vertical direction until dissipated from dissolution.

\section{A. Bubble Generator}

The bubble size distribution can be obtained from various experimental data sets [27] where the bubble population was 
directly measured either via acoustical and optical methods, a feature common to all can be found, namely: the spectra involves a considerable bubble population with radius between $1 \mathrm{~mm}$ and $6 \mathrm{~mm}$, which is supported by acoustical experimental data [27]. Veloso [13] gives a probable spectra location a radius of several $\mathrm{mm}$, but also states that their observations would not be inconsistent with a somewhat lower value.

Based on these arguments, we adopt the bubble size distribution from a gas emission depicted as chi-square distribution [28],

$$
f(x, k)= \begin{cases}\frac{x^{k-1} e^{-\frac{x^{2}}{2}}}{2^{\frac{k}{2}-1} \Gamma\left(\frac{k}{2}\right)}, & \text { if } x \geq 0 \\ 0, & \text { otherwise }\end{cases}
$$

where $x$ is the bubble radius, $k$ is known as "degrees of freedom", and $\Gamma(\cdot)$ is the gamma function. The distribution presents a dominant bubble size of around $2.5 \mathrm{~mm}$, as shown in Fig. 2.

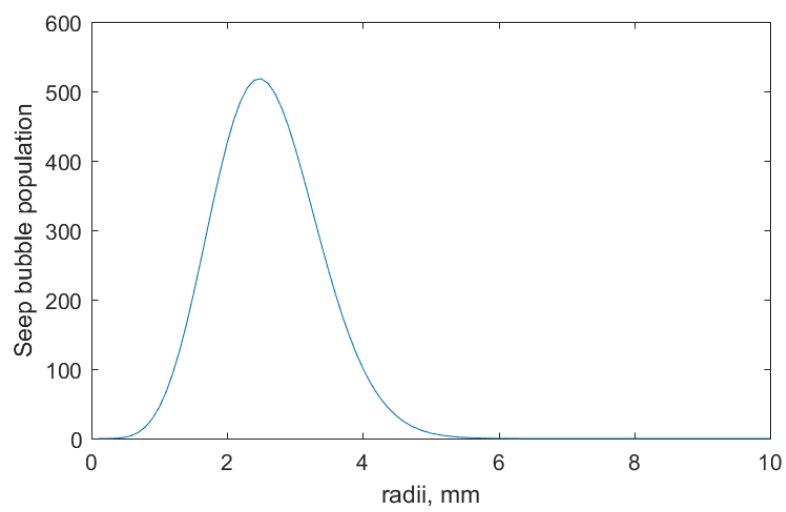

Fig. 2: Probability density function (PDF) of bubble size distribution of the gas emission. In this example, the dominant bubble size of around $2.5 \mathrm{~mm}$ and a gas flow rate of $5 \mathrm{~L} / \mathrm{min}$ is used.

With the bubble radius distribution $p_{b}=f(x, k)$, the mean volume $V_{m}$ across the bubble population is computed [29], and the average number of bubbles in the gas release experiment is then calculated as:

$$
N_{b}=V_{r} / V_{m}
$$

where $V_{r}\left[\mathrm{~m}^{3} / \mathrm{s}\right]$ is the gas flow rate, at which plumes generated can be taken from empirical data $(0.1-78 \mathrm{~L} / \mathrm{min}$ [8], [12], [13], [25], [30]-[33]). Assume the probability of the $n$th generated bubble is a random value $0 \leq P(n) \leq 1$, then the $n$th bubble equilibrium radius $R_{0}(n)$ can be obtained by the linear interpolation.

Reviewing various experimental data sets [13] where bubbles are unevenly generated in a range, common to all can be defined: the bubbles are generated randomly inside a circle at the seafloor. For a realistic parameterization of the gas plume, it is necessary to set the manner in which the plume possesses a horizontal area at the seafloor. The horizontal area is assumed as a circle, possessing a radius $\widehat{R}$.

\section{B. Single bubble transport mechanism}

As a bubble leaves the seabed and moves upward towards the sea surface, a few key environmental parameters were found to be affecting the bubble's transport and movement [27]. The bubble mass flux at time $t$, equalling to the change in the molar constant $N_{m o}$, is given by [27]

$$
\frac{\partial N_{m o}}{\partial t}=k_{B u b}(t) 4 \pi r(t)^{2}\left(C-P_{B}(t) / H\right),
$$

where $r(t)$ is the bubble equivalent spherical radius, $C$ is the aqueous concentration, $H$ is the Henry's law constant, $P_{B}(t)$ is the internal bubble pressure, and $k_{B u b}(t)$ is the bubble gas transfer velocity. The $P_{B}$ is defined as [27]

$$
P_{B}(t)=P_{A}(t)+\rho_{w} g z(t)+2 \sigma / r(t)
$$

where $\rho_{w} g z$ is the hydrostatic pressure, $\rho_{w}$ is the water density, $g$ is the gravitational constant, $z(t)=d_{\text {sea }}+h(t)$ is the bubble depth, $d_{\text {sea }}$ is the seafloor depth, $h(t)$ is the bubble height relative to the seafloor at time $t$, and $2 \sigma / r$ is the Laplace pressure due to surface tension $\sigma$. For clean, spherical bubbles with a thin concentration boundary layer, the $k_{B u b}(t)$ is given by [34]

$$
k_{B u b}(t)=\left\{0.212 \frac{D V_{B}(t)}{r(t)}\right\}^{0.5}
$$

where $D$ is the diffusion coefficient in $\mathrm{cm}^{2} \mathrm{~s}^{-2}$, and $V_{B}$ is the bubble rise velocity. For bubbles with a well developed boundary layer, the $k_{B u b}(t)$ can be given by [34]

$$
k_{B u b}(t)=\left\{\frac{2}{\pi}\left(1-2.89 R e(t)^{-0.5}\right) \frac{D V_{B}(t)}{r(t)}\right\}^{0.5},
$$

where $R e$ is the Reynolds number

$$
R e(t)=2 r(t) V_{B}(t) / v,
$$

where $v=1.4 \times 10^{-6} \mathrm{~m}^{2} / \mathrm{s}$ is the Kinematic viscosity. According to the ideal gas law $P_{B} V=N R T$ that converts moles into atmospheres, the change of bubble radius $\partial r(t)$ at time $t$ is derived as [27]

$\partial r(t)=r(t)\left(q \frac{\partial N}{\partial t}-\rho_{W} g \frac{\partial z}{\partial t}\right)\left(3\left(P_{A}+\rho_{W} g z\right)+\frac{4 \sigma}{r}\right)^{-1} \partial t$,

depending on which a bubble grows or shrinks as it moves upwards. For each time $t$, we update the radius $r$ as

$$
r(t+\Delta t)=r(t)+\partial r(t)
$$

The bubble rise velocity $V_{B}$ at time $t$ is given by

$$
\begin{aligned}
& V_{B}(t)= \\
& \left\{\left(\frac{\rho_{w} g r(t)^{2}}{3.68 M^{-0.038} \mu_{B}}\right)^{-d}\left(\frac{c \sigma}{\rho_{w} r(t)}+g r(t)\right)^{-d / 2}\right\}^{-(1 / d)},
\end{aligned}
$$

where $c$ and $d$ are empirical coefficients ( $c$ is either 1.2 for fresh water or 1.4 for sea water, and $d$ varies between 0.8 
(dirty) and 1.6 (clean)), $\mu_{B}$ is the bulk viscosity, and $\mathrm{M}$ is the dimensionless Morton number, defined as

$$
M=g \mu_{B}^{4} \rho_{w}^{-1} \sigma^{-3},
$$

which is solely dependent upon the liquid's physical properties. The height of a bubble at time $t$ is computed as

$$
h(t+\Delta t)=h(t)+\frac{V_{B}(t)+V_{B}(t+\Delta t)}{2} \partial t,
$$

where the initial $h(0)=0 \mathrm{~m}$ (seabed).

\section{Bubble plume updater}

As a bubble moves upward, it dissolves into the water. While the surface tension pressure results in faster dissolution of very small bubbles [35]. Bubbles will continue to shrink until they become so small to be removed from the water column. Considering a radius threshold $\widehat{r}$ to remove vary small bubbles [10], [27], the $\widehat{r}$ can be set as $1 \mu \mathrm{m}$.

As the time goes on, new bubbles are generated and added to the plume. The new bubbles adopt the same transport mechanism as described in Section II-B. Thus, the plume is updated. In the model, the impact of dissolution imposes a height-dependent bubble spectrum. The radius $r(t)$ in Eq.(9) and the height $h(t)$ in Eq.(12) control the vertical extent of the plume axis. Consequently, its maximum height is directly related to the dissolution.

\section{UNDERWATER SOUND SPEED AND ATTENUATION - VARIABILITY IMPOSED BY BUBBLE PLUME}

To calculate the sound speed in the bubbly medium and the attenuation introduced by the oscillating bubbles, we have chosen an approach based on the compressibility of the mixture [26], which renders an effective sound speed for linear pressure waves in bubbly liquids. The presence of oscillating bubbles causes the medium to be dispersive.

The gas flow rate determines the void fraction of the bubble medium at the seafloor. To simplify the use of the model developed herein, the dependence of void fraction on plume range at the seafloor have been fitted with the expression

$$
V_{g}=\sum_{i=1}^{n} v_{i}
$$

where $v(i)$ is the volume of bubbles with the $i$ th radius. The average void fraction at height $t$ for the gas plume can be calculated from

$$
V_{o}=\frac{V_{g}}{\pi r^{2}}
$$

which holds for circular distribution of bubble generation at the seafloor.

The effective sound speed in the medium can be determined by calculating the change in compressibility $(\Delta K)$ introduced by the gas in bubble form with due regard to its resonant characteristics. This is accomplished by calculating the compressibility of a single bubble and integrating over the distribution of bubble sizes. Interaction between bubbles is ignored. The resulting compressibility of the gas in bubble form $(\Delta K)$ is then inserted in Wood's equation [36] to obtain the effective sound speed in the mixture. The compressibility of the mixture is given by

$$
K=(1-V) K_{0}+\Delta K,
$$

where $K_{0}=1 /\left(\rho_{0} c^{2}\right)$ is the compressibility of the water in the absence of the bubbles and $V$ is the fractional volume occupied by bubbles. To compute the $\Delta K$, we adopt the expression from [26] for water containing $N$ bubbles per unit volume, each having radius $a$, and assuming $k a<1$. This expression can be rewritten as

$$
\Delta K(f, z)=\frac{N}{\rho_{0} \pi f^{2}} \frac{a}{\left(\left(\frac{f_{r}}{f}\right)^{2}-1+i \delta\right)},
$$

where $\delta$ is the damping constant and $f_{r}$ is the resonance frequency. The extension to a distribution of bubble radii yields

$$
\Delta K(f, z)=\frac{1}{\rho_{0} \pi f^{2}} \int_{a_{\min }}^{a_{\max }}\left[\frac{a n(a)}{\left(\left(\frac{f_{r}}{f}\right)^{2}-1+i \delta\right)}\right] d a .
$$

The resonance frequency is calculated with due regard to thermal effects and the presence of surface tension. Under the assumption of adiabatic oscillations, and neglecting viscous effects, the resonance frequency $[\mathrm{Hz}]$ for a bubble radius $a$ [ $\mu \mathrm{m}]$ is given by the Minnaert resonance [37], [38]

$$
f_{r}=\frac{1}{2 \pi a}\left(\frac{3 \gamma p_{A}}{\rho_{0}}\right)^{2},
$$

where $a$ is the radius of the bubble, $\gamma$ is the polytropic coefficient, $p_{A}$ is the ambient pressure, and $\rho_{0}$ is the density of seawater. The density of the bubbly mixture (effective medium) is given by

$$
\rho_{\mathrm{eff}}=(1-V) \rho_{0}+V \rho_{\mathrm{gas}},
$$

and the effective sound speed is

$$
c_{\mathrm{eff}}^{-2}=\left[(1-V) \rho_{0}+V \rho_{\mathrm{gas}}\right]\left[(1-V) K_{0}+\Delta K\right] .
$$

Since $\Delta K$ is a complex number, the effective sound speed is complex; the real part $\left(c_{b}\right)$ provides the phase velocity and the imaginary part yields the attenuation due to the bubbles

$$
\begin{aligned}
c_{b} & =\operatorname{Re}\left\{c_{\text {eff }}\right\}, \\
\alpha_{b}\left(\frac{\mathrm{dB}}{\mathrm{m}}\right) & =\frac{20}{\ln (10)} \operatorname{Im}\left\{\frac{1}{c_{\mathrm{eff}}}\right\} .
\end{aligned}
$$

For low void fractions $\left(V \gg \rho_{\text {gas }} / \rho_{0}\right)$, Eq.(20) can be simplified to

$$
c_{\mathrm{eff}}^{-2}=c_{0}^{-2}+\rho_{0} \Delta K .
$$

When $\Delta K$ from Eq.(17) is replaced in Eq.(20)

$$
c_{\mathrm{eff}}^{-2}=c_{0}^{-2}+\frac{1}{\pi f^{2}} \int_{a_{\min }}^{a_{\max }}\left[\frac{a n(a)}{\left(\left(\frac{f_{r}}{f}\right)^{2}-1+i \delta\right)}\right] d a .
$$

It should be noted that, in this case of low void fraction, this result agrees with that of Commander and Prosperetti [39] which was derived on a more rigorous basis and has been shown to match experimental data quite well. For the numerical calculations in this work, the full Eq.(20) was implemented. 
We note that multiple scattering among bubbles is ignored in both of these approaches. As shown by Feulliade [40], for a broad distribution of radii with no sharp peak, this assumption is safe at the void fractions involved in the present modelling $\left(V<10^{-4}\right)$. Since in the present study the bubble spectra are truncated at $1000 \mu \mathrm{m}$, the condition $k a<1$ places an upper limit on the frequency for which Eq.(17) is reliable. This upper limit is about $240 \mathrm{kHz}$.

\section{NUMERICAL RESULTS}

To illustrate the dispersion associate with the different spectra parameterized in this work, calculations of the sound speed and attenuation will be carried out for an assumed gas flow rate equal to $5 \mathrm{~L} / \mathrm{min}$ and evaluated at the seafloor $(120 \mathrm{~m}$ depth). These are the parameters that were used to generate the bubble distributions shown in Fig. 2.

In the example that follows $\mathrm{CO}_{2}$ gas is used and, the effective sound speed and attenuation induced by the presence of bubble plumes are calculated for an otherwise lossless isovelocity half-space $\left(c_{0}=1500 \mathrm{~m} / \mathrm{s}\right)$. The acoustic frequency is from $1 \mathrm{kHz}$ to $9 \mathrm{kHz}$. The spatial increments used in the model to specify the height-dependent bubble spectrum are $\Delta z=0.1 \mathrm{~m}$ for $\mathrm{CO}_{2}$.

Since images of seabed emitted gas plumes derived or photographed from measurements have been presented in the literature [25], [31], [41], [42], it is appropriate, therefore, to try and synthesize similar images using the model developed and to calculate sound speed and attenuation. Fig. 3 shows two-dimensions (2-D) simulated images of physical gaseous $\mathrm{CO}_{2}$ bubble plumes.

\section{A. Gaseous $\mathrm{CO}_{2}$ emission}

The largest bubbles are found close to the modelled seabed $(0.1 \mathrm{~m}$ in Fig. 4(a)). As the height above the seabed increases, these large bubbles become increasingly rare $(3.0 \mathrm{~m}$ in Fig. 4(b)), due to the dissolution. This leads to higher resonance frequencies at $3.0 \mathrm{~m}$ (Fig. 5(b)) than that at $0.1 \mathrm{~m}$ (Fig. 5(a)). For these cases, the average void fraction (Fig. 6) is $1.7 \times 10^{-5}$ at $0.1 \mathrm{~m}$ and $0.5 \times 10^{-6}$ at $3.0 \mathrm{~m}$ where the smaller bubbles dominate.

\section{B. Fluctuations of sound speed and attenuation}

According to Eq.(23) and Eq.(21b), as the frequency increases, the effective average sound speed (Fig. 7) and attenuation (Fig. 8) are frequency dependent and change both at heights of $0.1 \mathrm{~m}$ and $3.0 \mathrm{~m}$ for an acoustic wave travelling through the bubbly medium. At the height of $0.1 \mathrm{~m} / \mathrm{s}$, Fig. 7(a) shows that the sound speed decreases from $1485 \mathrm{~m} / \mathrm{s}$ at very low frequencies to the lowest sound speed $\sim 1450 \mathrm{~m} / \mathrm{s}$ at frequency $\sim 2.5 \mathrm{kHz}$; after that, it increases to the highest sound speed $\sim 1520 \mathrm{~m} / \mathrm{s}$ at frequency $\sim 5 \mathrm{kHz}$, and finally tends to the normal sound speed $1500 \mathrm{~m} / \mathrm{s}$ at higher frequencies.

Fig. 8(a) shows the attenuation perturbation at $0.1 \mathrm{~m}$ height, where the attenuation increases from low frequencies to the highest point at $\sim 4 \mathrm{kHz}$ where the attenuation attains high values, followed by a decreasing attenuation towards higher

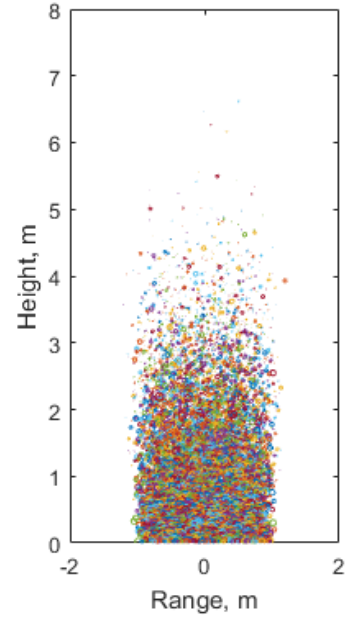

Fig. 3: 2-D $\mathrm{CO}_{2}$ gas bubble plume shape. Flow rate $5 \mathrm{~L} / \mathrm{min}$. Colors are used for differentiating different single bubbles.

frequencies. At the height of $3.0 \mathrm{~m}$, Fig. 7(b) and Fig. 8(b) show similar trends of sound speed perturbation as the frequency increases. While due to less bubbles and the dominant smaller bubbles at this height $(3 \mathrm{~m})$, the lowest and highest sound speeds and the highest attenuation are not as significant as that close to the seabed $(0.1 \mathrm{~m})$. Also, as higher frequencies are closer to resonant frequency of smaller bubbles while smaller bubbles are depleted less rapidly with height, the variation of sound speed and attenuation is not significant at higher frequencies. Further, more dispersed bubble size distribution at $3.0 \mathrm{~m}$ than that at $0.1 \mathrm{~m}$ makes the higher variation of sound speed and attenuation.

To illustrate the height dependence of the perturbation within the plume, Fig. 9 and Fig. 10 show results for frequencies of $1 \mathrm{kHz}, 3 \mathrm{kHz}, 6 \mathrm{kHz}$ and $9 \mathrm{kHz}$. As expected, the presence of the bubbles are sensed in deeper water (close to the seafloor) than in shallow water.

The height dependence of the perturbation in the sound speed $\left(\Delta c=c_{b}-c_{0}\right.$, where $c_{0}$ is the sound speed in the water without the bubbles) that is introduced by the presence of the bubble clouds as shown in Fig. 9. In Fig. 9(a), a single contour line, showing changes of $-60 \mathrm{~m} / \mathrm{s}$ in the sound speed at frequency $1 \mathrm{kHz}$, traces the shape of the gas plumes (Fig. 3), and Fig. 9(b), (c) and (d) show contours for $-200 \mathrm{~m} / \mathrm{s}$, $60 \mathrm{~m} / \mathrm{s}$ and $20 \mathrm{~m} / \mathrm{s}$ at frequencies $3 \mathrm{kHz}, 6 \mathrm{kHz}$ and $9 \mathrm{kHz}$, respectively. It is clear from these figures that the sound speed perturbation is significant close to the seafloor. Inside the gas plume the perturbed sound speed exceeds the unperturbed by about $200 \mathrm{~m} / \mathrm{s}$ at the seafloor. As can be inferred from inspection of the dispersion curves (Fig. 7), the sound speed perturbation coefficient at $1 \mathrm{kHz}$ is not much different from that for $6 \mathrm{kHz}$ since both frequencies lie nearly equidistant to either side of the sound speed perturbation peak. In each case, the frequencies having resonance at radii corresponding to the breaking points in the bubble spectra are indicated. 


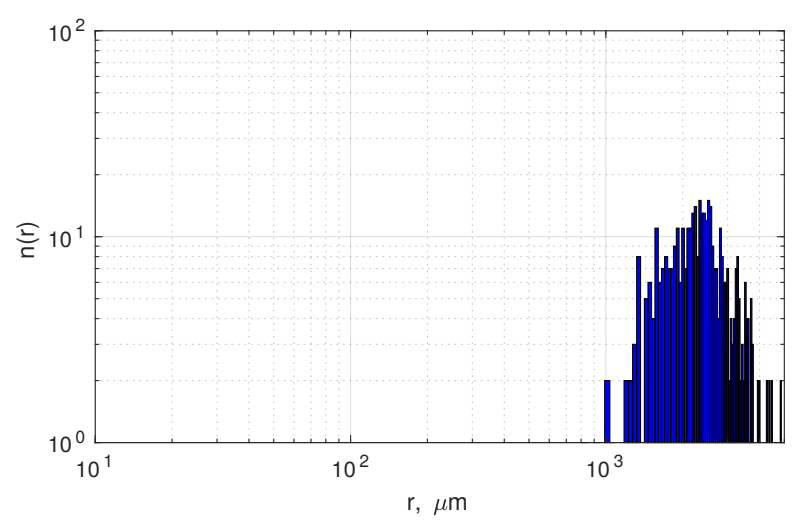

(a) $0.1 \mathrm{~m}$.

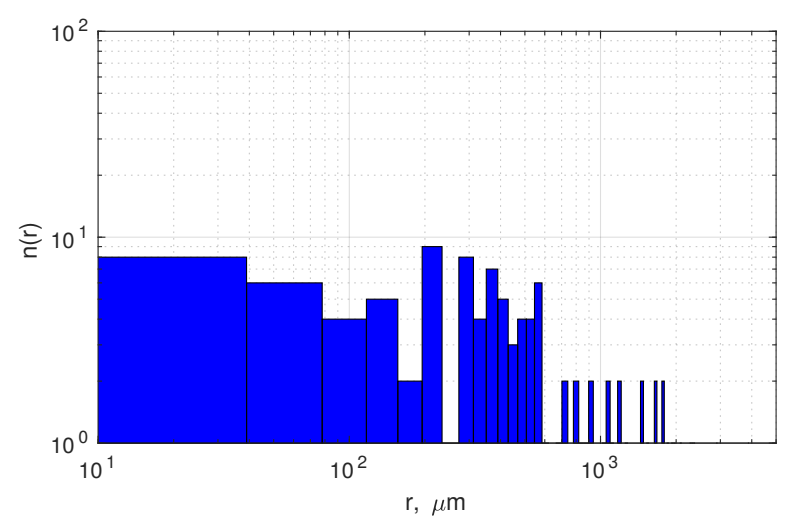

(b) $3.0 \mathrm{~m}$.

Fig. 4: Bubble population at different heights. $\mathrm{CO}_{2}$ gas flow rate $5 \mathrm{~L} / \mathrm{min}$. (a) $0.1 \mathrm{~m}$. (b) $3.0 \mathrm{~m}$.

The bubble-induced attenuation field (chemical attenuation not included) at frequency $1 \mathrm{kHz}$ is plotted in Fig. 10(a), showing the $0.01 \mathrm{~dB} / \mathrm{m}$ contour, traces the shape of the gas plume (Fig. 3), and Fig. 10(b), (c) and (d) show contours for $18 \mathrm{~dB} / \mathrm{m}, 10 \mathrm{~dB} / \mathrm{m}$ and $3.5 \mathrm{~dB} / \mathrm{m}$ at frequencies $3 \mathrm{kHz}, 6 \mathrm{kHz}$ and $9 \mathrm{kHz}$, respectively. It is clear from these figures that the attenuation is significant close to the seafloor. Comparing Fig. 9(d) and Fig. 10(d), it can be noted that at this moderately high frequency, even at heights where the sound speed perturbation is small, the attenuation is significant. The four preceding examples show that the presence of bubble plumes induce significant range-dependent changes in both the sound speed and attenuation.

Fig. 11(a) and (b) show the height dependence of the sound speed and attenuation along the axis of the gas plume induced by the presence of the bubble at the four frequencies, respectively. The perturbed sound speed exceeds that of the bubble-free medium (assuming $c_{0}=1500 \mathrm{~m} / \mathrm{s}$ ) at frequencies of $6 \mathrm{kHz}$ and $9 \mathrm{kHz}$ and beneath it at $1 \mathrm{kHz}$ and $3 \mathrm{kHz}$, as expected by inspection of the contour shown in Fig. 9. The perturbed attenuation exceeds that of the bubble-free medium (assuming $0 \mathrm{~dB} / \mathrm{m}$ ) at frequencies of $3 \mathrm{kHz}, 6 \mathrm{kHz}$ and $9 \mathrm{kHz}$ and can hardly be observed at frequency $1 \mathrm{kHz}$, as expected

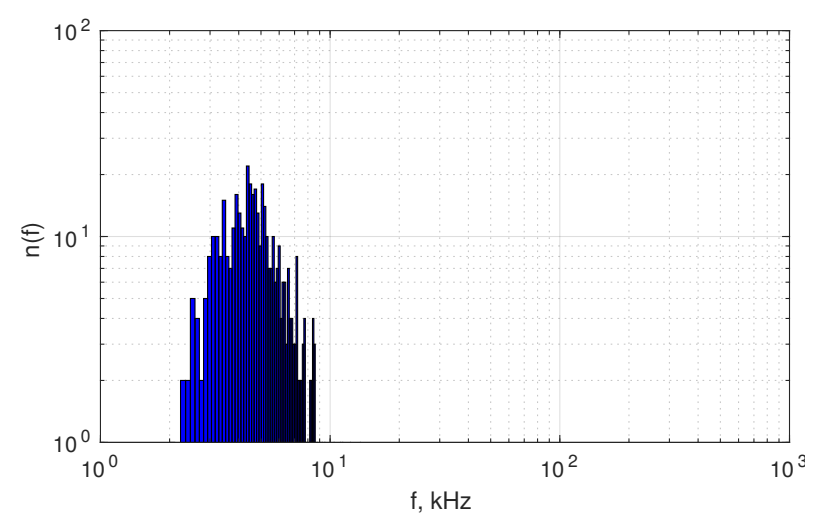

(a) $0.1 \mathrm{~m}$.

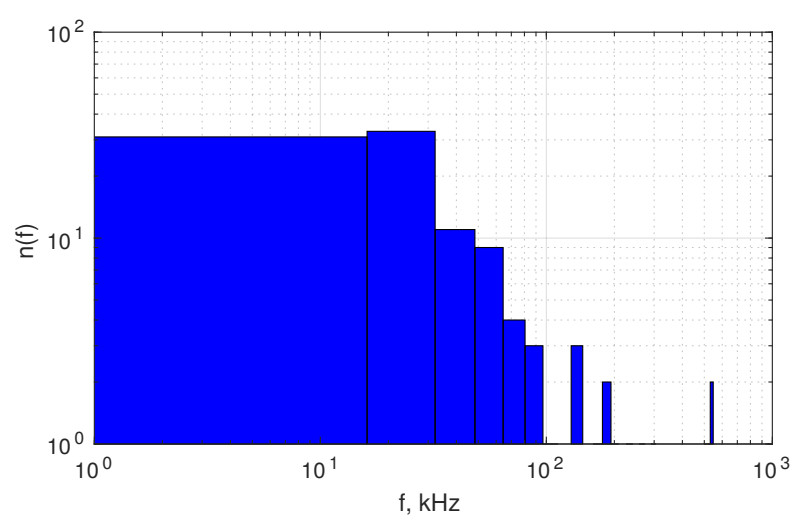

(b) $3.0 \mathrm{~m}$.

Fig. 5: Resonance frequency at different heights above the seabed. $\mathrm{CO}_{2}$ gas flow rate $5 \mathrm{~L} / \mathrm{min}$. (a) $0.1 \mathrm{~m}$. (b) $3.0 \mathrm{~m}$.

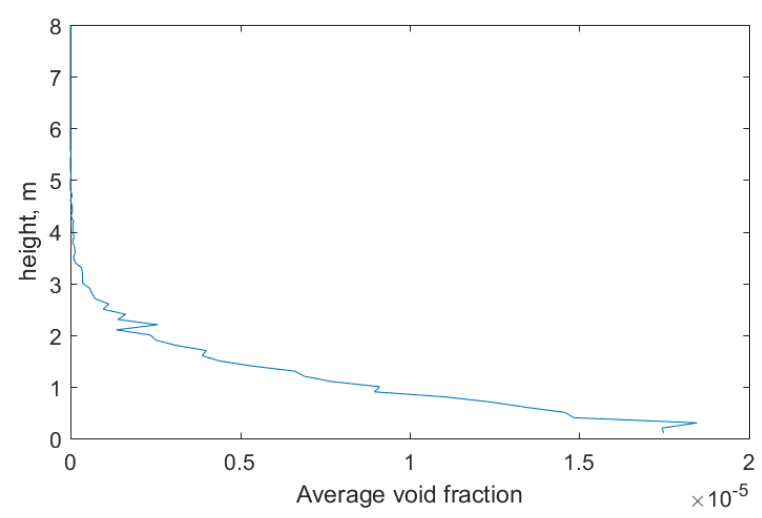

Fig. 6: Average void fraction at different heights above the seabed. $\mathrm{CO}_{2}$ gas flow rate $5 \mathrm{~L} / \mathrm{min}$.

by inspection of the contour shown in Fig. 10.

It should be pointed out that the calculation of the complex sound speed for an acoustic frequency between the resonance frequencies associated with the largest and smallest bubbles is strongly dependent on the spectral slope, the position of the spectral peak, and the limits of integration. For example, different spectral shapes (keeping the total void fraction 


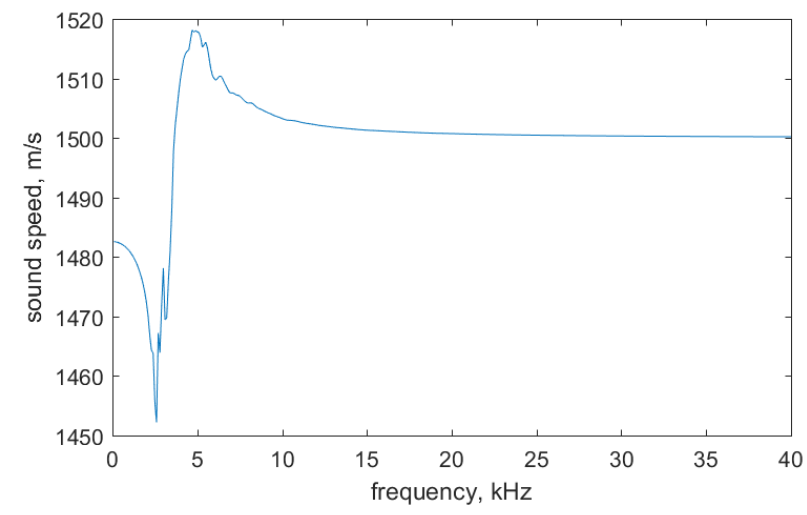

(a) $0.1 \mathrm{~m}$.

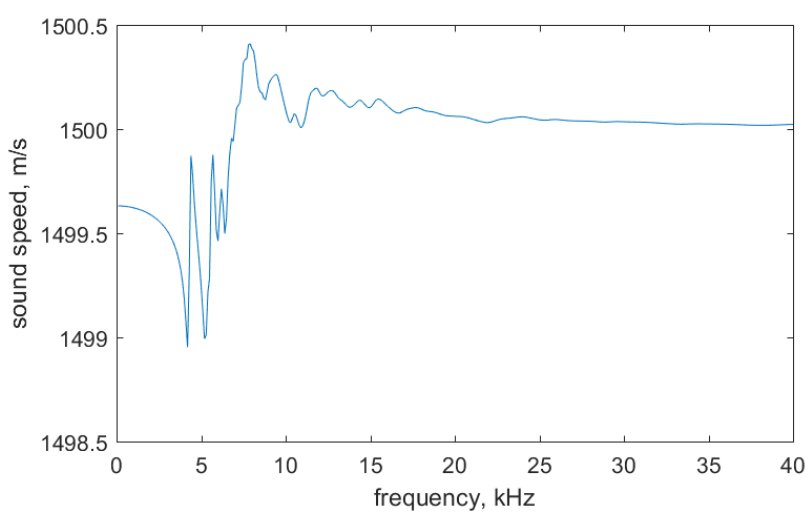

(b) $3.0 \mathrm{~m}$.

Fig. 7: Variation of sound speed at different heights above the seabed. $\mathrm{CO}_{2}$ gas flow rate $5 \mathrm{~L} / \mathrm{min}$. (a) $0.1 \mathrm{~m}$. (b) $3.0 \mathrm{~m}$.

constant by modifying the constant $N_{0}$ ) will lead to different dispersion curves for the phase velocity and attenuation. On the other hand, at low frequencies (i.e., for frequencies below the resonance frequency of the largest bubbles), the oscillating behavior of the bubbles no longer plays a role and the sound speed becomes insensitive to the spectral shape. Under these conditions, the change to the real part of the sound speed can be estimated from the classical Wood's equation for the given void fraction and the change to the attenuation can be neglected.

This completes the parameterization of the plumes. As shown, considering the high variability of bubble plume that can be found at sea under roughly similar environmental conditions, the proposed model encompasses the measurements. Once the functional form for the intervening factors have been set, the constant has to be chosen such that it leads to a bubble density and void fraction (V) within the bounds set. At a certain height for the plume, it is shown that the horizontal extent of the plume should be a small fraction of what it is at the seafloor.

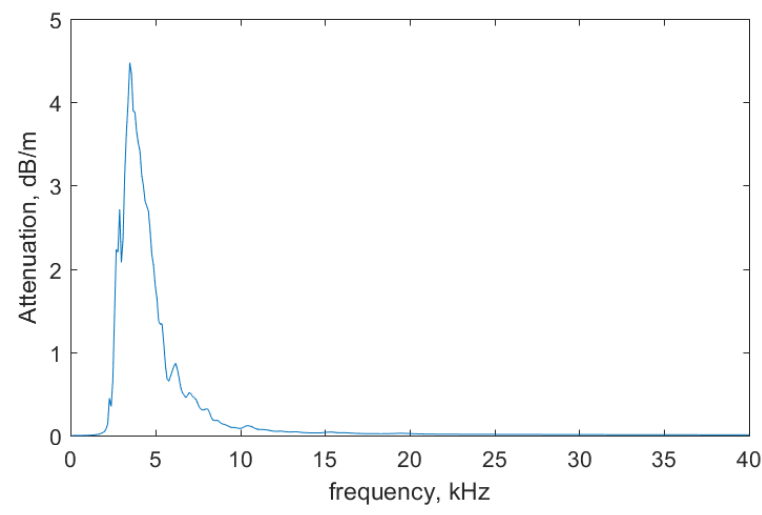

(a) $0.1 \mathrm{~m}$.

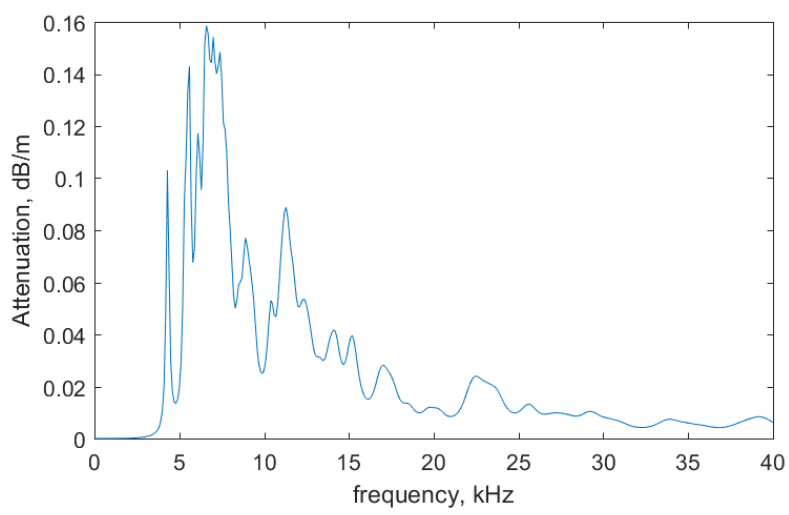

(b) $3.0 \mathrm{~m}$.

Fig. 8: Variation of attenuation at different heights above the seabed. $\mathrm{CO}_{2}$ gas flow rate $5 \mathrm{~L} / \mathrm{min}$. (a) $0.1 \mathrm{~m}$. (b) $3.0 \mathrm{~m}$.

\section{STATISTICAL FLUCTUATION OF THE SOUND SPEED AND ATTENUATION}

In the previous section, the change induced by the presence of bubble plume on the sound speed and attenuation was estimated in a deterministic manner. To simulate ocean experiments, it is necessary to bring in statistical variations. For example, within the gas plumes, the bubble concentration will change from plume to plume, the plume height will fluctuate, the size of the bubbles will change and the spacing between them will also be randomly distributed. There is not enough information in the literature to quantify all the statistical deviations necessary to make the model universally applicable. Nevertheless, large variations in the bubble density at a given height over very short periods of time have been observed [42], [43].

In the present model, the height of the plumes, as well as the width of the plumes, is controlled by the various parameters. The average and the maximum plume height is independent to the flow rate. Also, from the direct sound speed data of [44], variations in the measured sound speed time series on the order of $10 \%$ and larger are observed. As a guide to estimate the variation in the production of bubbles within gas plumes, we 


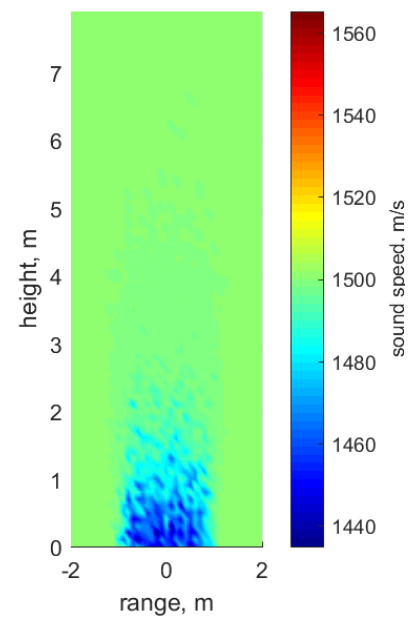

(a) $1 \mathrm{kHz}$.

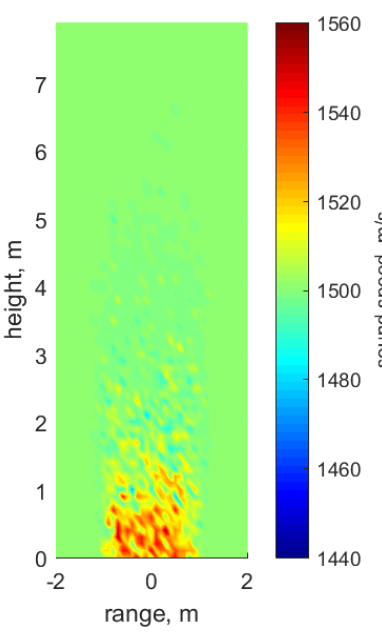

(c) $6 \mathrm{kHz}$

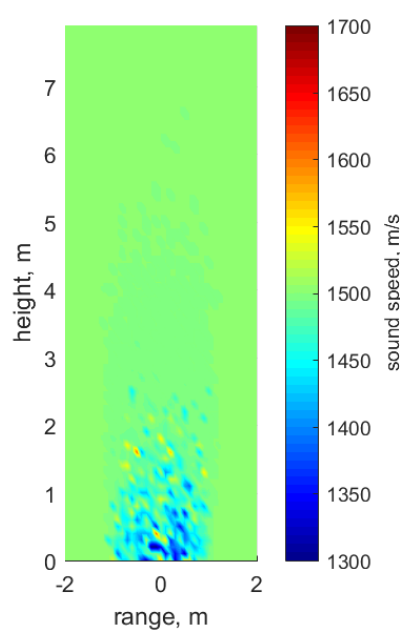

(b) $3 \mathrm{kHz}$.

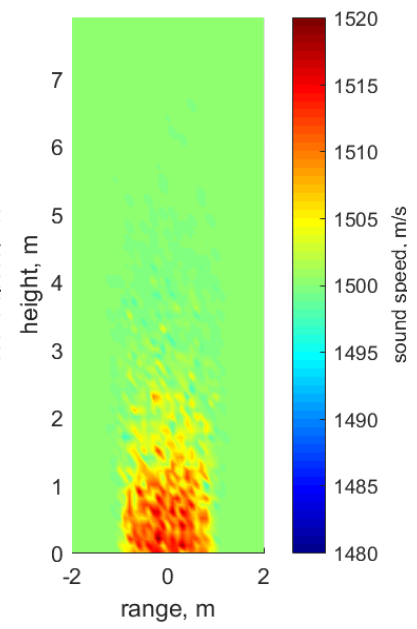

(d) $9 \mathrm{kHz}$.
Fig. 9: Variation of sound speed at various frequencies. $\mathrm{CO}_{2}$ gas flow rate $5 \mathrm{~L} / \mathrm{min}$. (a) $1 \mathrm{kHz}$. (b) $3 \mathrm{kHz}$. (c) $6 \mathrm{kHz}$. (d) $9 \mathrm{kHz}$.

have resorted to an expression derived by [45] which provides the bubble density.

\section{CONCLUSIONS AND DISCUSSION}

In this paper, we utilized an existing scheme for generation of gas plumes and, developed an active acoustic model that predicts sound speed and attenuation perturbations from seabed gas emissions at different frequencies of insonification, and at different heights above the seabed. Due to the disparity of data available on seabed gas emissions and the many intervening parameters, it is difficult, if not impossible, to develop a single generally applicable model for use on seabed gas emissions in underwater acoustics. Future work will be focused on the lab and field experiments to confirm the effectiveness of the developed model, and will extend it to other greenhouse gases, e.g., $\mathrm{CH}_{4}$ leakage at the seabed.

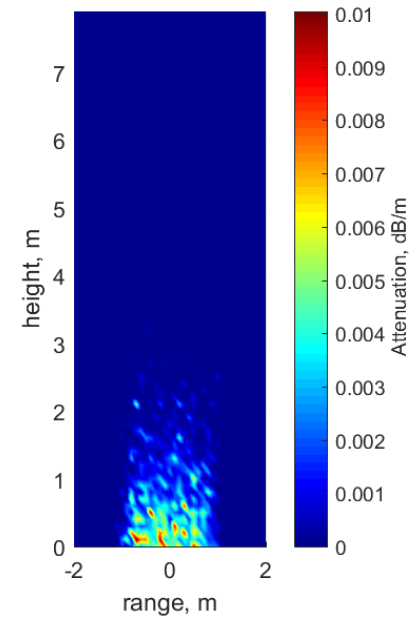

(a) $1 \mathrm{kHz}$.

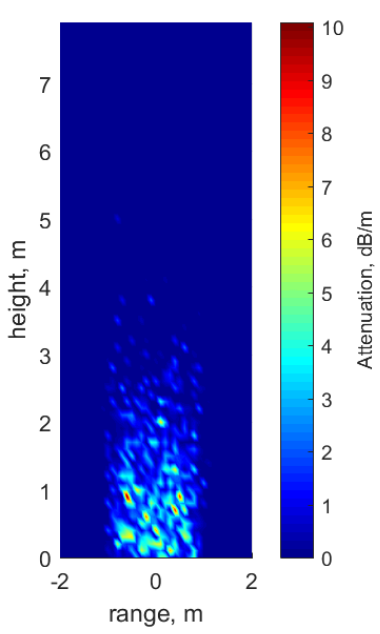

(c) $6 \mathrm{kHz}$.

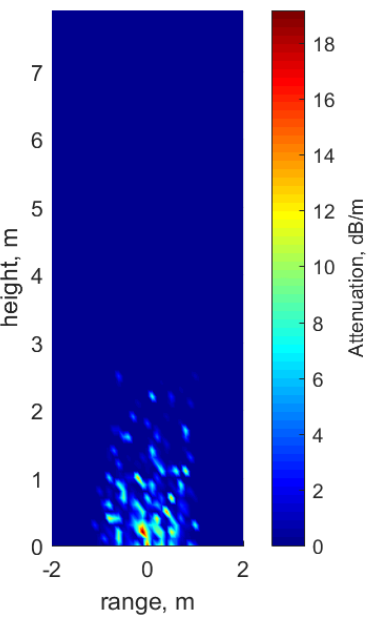

(b) $3 \mathrm{kHz}$.

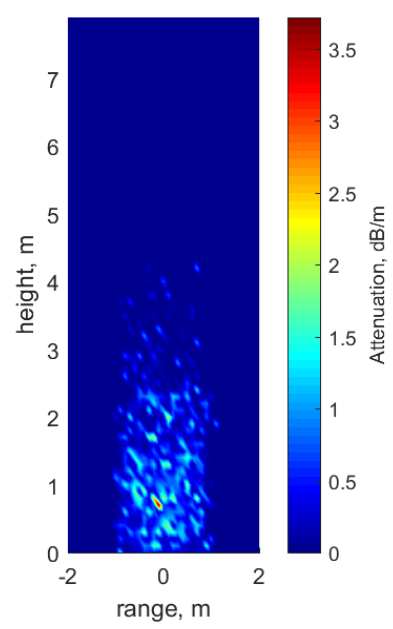

(d) $9 \mathrm{kHz}$
Fig. 10: Variation of attenuation at various frequencies. $\mathrm{CO}_{2}$ gas flow rate $5 \mathrm{~L} / \mathrm{min}$. (a) $1 \mathrm{kHz}$. (b) $3 \mathrm{kHz}$. (c) $6 \mathrm{kHz}$. (d) $9 \mathrm{kHz}$.

\section{ACKNOWLEDGEMENT}

Funding for this work was provided by the European Unions Horizon 2020 research and innovation programme under the grant agreement number 654462 (STEMM-CCS).

\section{REFERENCES}

[1] H. K. Hvidevold, G. Alendal, T. Johannessen, and A. Ali, "Survey strategies to quantify and optimize detecting probability of a co 2 seep in a varying marine environment," Environmental Modelling \& Software, vol. 83, pp. 303-309, 2016.

[2] P. Taylor, H. Stahl, M. E. Vardy, J. M. Bull, M. Akhurst, C. Hauton, R. H James, A. Lichtschlag, D. Long, D. Aleynik et al., "A novel sub-seabed co 2 release experiment informing monitoring and impact assessment for geological carbon storage," International Journal of Greenhouse Gas Control, vol. 38, pp. 3-17, 2015.

[3] D. Atamanchuk, A. Tengberg, D. Aleynik, P. Fietzek, K. Shitashima, A. Lichtschlag, P. O. Hall, and H. Stahl, "Detection of co2 leakage from a simulated sub-seabed storage site using three different types of pco 2 


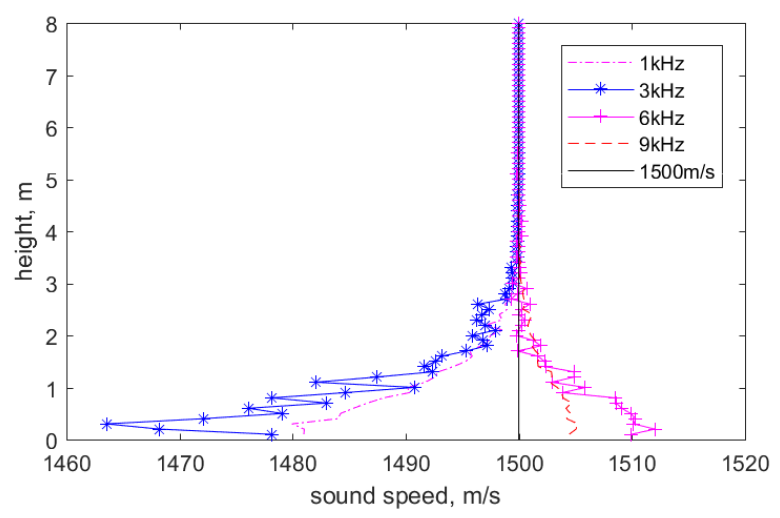

(a) Sound Speed.

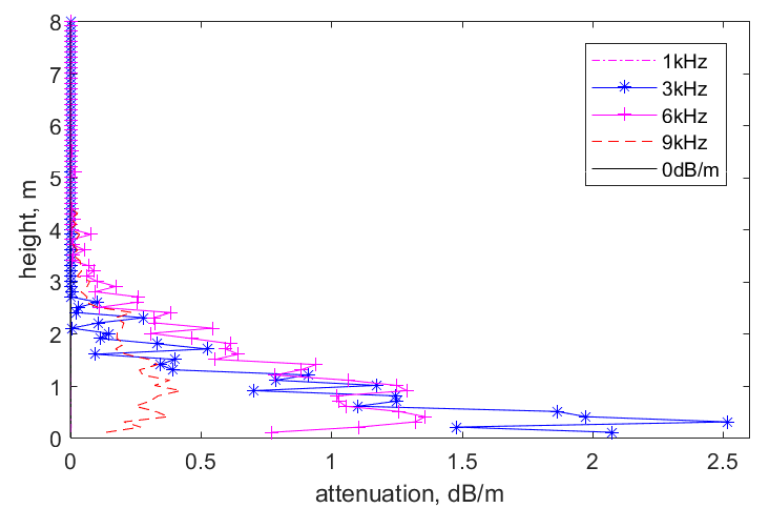

(b) Attenuation.

Fig. 11: Variations of sound speed and attenuation at different frequencies. $\mathrm{CO}_{2}$ gas flow rate $5 \mathrm{~L} / \mathrm{min}$. (a) Sound Speed. (b) Attenuation.

sensors," International Journal of Greenhouse Gas Control, vol. 38, pp. $121-134,2015$.

[4] M. Cevatoglu, J. M. Bull, M. E. Vardy, T. M. Gernon, I. C. Wright, and D. Long, "Gas migration pathways, controlling mechanisms and changes in sediment acoustic properties observed in a controlled sub-seabed co 2 release experiment," International Journal of Greenhouse Gas Control, vol. 38, pp. 26-43, 2015.

[5] Y. Kano and T. Sato, "Gas-liquid-Solid Three-phase Simulation on CO2 Seeping through Marine Sediment," Energy Procedia, vol. 114, pp. 3385-3392, 2017

[6] E. Rastelli, C. Corinaldesi, A. DellAnno, T. Amaro, A. M. Queirós, S. Widdicombe, and R. Danovaro, "Impact of $\mathrm{CO} 2$ leakage from subseabed carbon dioxide capture and storage (CCS) reservoirs on benthic virus-prokaryote interactions and functions," Frontiers in microbiology, vol. $6,2015$.

[7] N. Koukouzas, P. Lymperopoulos, A. Tasianas, and S. Shariatipour "Feasibility Study for The Setting Up of a Safety System for Monitoring CO2 Storage at Prinos Field, Greece," in IOP Conference Series: Earth and Environmental Science, vol. 44, no. 5. IOP Publishing, 2016, p. 052043.

[8] J. Blackford, H. Stahl, J. M. Bull et al., "Detection and impacts of leakage from sub-seafloor deep geological carbon dioxide storage," Nature Climate Change, vol. 4, pp. 1011-1016, 2014.

[9] J. Li, P. R. White, J. M. Bull, and T. G. Leighton, "A noise impact assessment model for passive acoustic measurements of seabed gas fluxes," Ocean Engineering, vol. 183, no. 1, pp. 294-304, 2019.

[10] T. Leighton and P. White, "Quantification of undersea gas leaks from carbon capture and storage facilities, from pipelines and from methane seeps, by their acoustic emissions," in Proc. R. Soc. A, vol. 468, no.
2138. The Royal Society, 2012, pp. 485-510.

[11] S. O'hara, P. Dando, U. Schuster, A. Bennis, J. Boyle, F. Chui, T. Hatherell, S. Niven, and L. Taylor, "Gas seep induced interstitial water circulation: observations and environmental implications," Continental Shelf Research, vol. 15, no. 8, pp. 931-948, 1995.

[12] K. Thomanek, O. Zielinski, H. Sahling, and G. Bohrmann, "Automated gas bubble imaging at sea floor-a new method of in situ gas flux quantification," Ocean Science, vol. 6, no. 2, pp. 549-562, 2010.

[13] M. Veloso, J. Greinert, J. Mienert, and M. De Batist, "A new methodology for quantifying bubble flow rates in deep water using splitbeam echosounders: Examples from the arctic offshore nw-svalbard," Limnology and Oceanography: Methods, vol. 13, no. 6, pp. 267-287, 2015.

[14] J. Li, L. Liao, and Y. V. Zakharov, "Space-time cluster combining for UWA communications," in IEEE OCEANS 2016-Shanghai, 2016, pp. $1-6$.

[15] J. Li and Y. V. Zakharov, "Efficient use of space-time clustering for underwater acoustic communications," IEEE Journal of Oceanic Engineering, vol. 43, no. 1, pp. 173-183, 2018.

[16] J. Li, Y. V. Zakharov, and B. Henson, "Multibranch Autocorrelation Method for Doppler Estimation in Underwater Acoustic Channels," IEEE Journal of Oceanic Engineering, vol. 43, no. 4, pp. 1099 - 1113 , 2018.

[17] J. Li and Y. V. Zakharov, "Sliding-window homotopy adaptive filter for estimation of sparse UWA channels," in IEEE 9th Sensor Array and Multichannel Signal Processing Workshop (SAM), Rio de Janeiro, Brazil, 2016, pp. 1-4.

[18] J. Li, "DOA tracking in time-varying underwater acoustic communication channels," in MTS/IEEE OCEANS 2017-Aberdeen, UK, 2017, pp. $1-9$.

[19] J. Li and Y. V. Zakharov, "Sliding window adaptive filter with diagonal loading for estimation of sparse UWA channels," in IEEE OCEANS 2016-Shanghai, China, 2016, pp. 1-5.

[20] F. Yuan, Z. Jia, J. Li, and E. Cheng, "STLFM Signal Based Adaptive Synchronization for Underwater Acoustic Communications," IEEE Access, vol. 7, pp. 28 734-28 748, 2019.

[21] Y. Zhang, T. Wu, Y. Zakharov, and J. Li, "MMP-DCD-CV based sparse channel estimation algorithm for underwater acoustic transform domain communication system," Applied Acoustics, vol. 154, pp. 43-52, 2019.

[22] Y. Zhang, J. Li, Y. Zakharov, X. Li, and J. Li, "Deep learning based underwater acoustic OFDM communications," Applied Acoustics, vol. 154, pp. 53-58, 2019.

[23] E. King, "An investigation into the effects of underwater bubble formations on sound speed and attenuation," The Plymouth Student Scientist, vol. 9, no. 1, pp. 105-144, 2016.

[24] I. Leifer and D. Culling, "Formation of seep bubble plumes in the coal oil point seep field," Geo-Marine Letters, vol. 30, no. 3-4, pp. 339-353, 2010.

[25] E. J. Sauter, S. I. Muyakshin, J.-L. Charlou, M. Schlüter, A. Boetius, K. Jerosch, E. Damm, J.-P. Foucher, and M. Klages, "Methane discharge from a deep-sea submarine mud volcano into the upper water column by gas hydrate-coated methane bubbles," Earth and Planetary Science Letters, vol. 243, no. 3, pp. 354-365, 2006.

[26] C. S. Clay and H. Medwin, "Acoustical oceanography: principles and applications," 1977.

[27] I. Leifer and R. K. Patro, "The bubble mechanism for methane transport from the shallow sea bed to the surface: A review and sensitivity study," Continental Shelf Research, vol. 22, no. 16, pp. 2409-2428, 2002.

[28] C.-H. Lee, L. Erickson, and L. Glasgow, "Bubble breakup and coalescence in turbulent gas-liquid dispersions," Chemical Engineering Communications, vol. 59, no. 1-6, pp. 65-84, 1987.

[29] K. Yeh and K. Kwan, "A comparison of numerical integrating algorithms by trapezoidal, lagrange, and spline approximation," Journal of Pharmacokinetics and Pharmacodynamics, vol. 6, no. 1, pp. 79-98, 1978.

[30] P. Linke, M. Haeckel, J. Schneider von Deimling, L. Vielstädte, M. Schmidt, J. Karstens, C. Berndt, H. Herreilers, A. Lichtschlag, R. James et al., Fluxes of $\mathrm{CO}_{2}$ from natural seep sites and Sleipner storage site. $\mathrm{ECO}_{2}$ Project Office, 2014, no. 265847. [Online]. Available: https://www.lombardiresearchgroup.com/files/fluxes_of_co2_from_ natural_seep_sites_and_sleipner_storage_site-05082015-143518.pdf

[31] I. Leifer and I. MacDonald, "Dynamics of the gas flux from shallow gas hydrate deposits: interaction between oily hydrate bubbles and the oceanic environment," Earth and Planetary Science Letters, vol. 210, no. 3, pp. 411-424, 2003. 
[32] M. Torres, J. McManus, D. Hammond, M. D. Angelis, K. Heeschen, S. Colbert, M. Tryon, K. Brown, and E. Suess, "Fluid and chemical fluxes in and out of sediments hosting methane hydrate deposits on Hydrate Ridge, OR, I: Hydrological provinces," Earth and Planetary Science Letters, vol. 201, no. 3, pp. 525-540, 2002.

[33] H. Sahling, G. Bohrmann, Y. G. Artemov, A. Bahr, M. Brüning, S. A. Klapp, I. Klaucke, E. Kozlova, A. Nikolovska, T. Pape et al., "Vodyanitskii mud volcano, sorokin trough, black sea: Geological characterization and quantification of gas bubble streams," Marine and Petroleum Geology, vol. 26, no. 9, pp. 1799-1811, 2009.

[34] R. Clift, J. Grace, and M. Weber, "Bubbles, drops and particles," Academic Press, New York, p. 380, 1978.

[35] J. C. Novarini, R. S. Keiffer, and G. V. Norton, "A model for variations in the range and depth dependence of the sound speed and attenuation induced by bubble clouds under wind-driven sea surfaces," IEEE journal of oceanic engineering, vol. 23, no. 4, pp. 423-438, 1998.

[36] E. Lamarre and W. Melville, "Void-fraction measurements and soundspeed fields in bubble plumes generated by breaking waves," The Journal of the Acoustical Society of America, vol. 95, no. 3, pp. 1317-1328, 1994.

[37] M. Minnaert, "Xvi. on musical air-bubbles and the sounds of running water," The London, Edinburgh, and Dublin Philosophical Magazine and Journal of Science, vol. 16, no. 104, pp. 235-248, 1933.

[38] P. A. Hwang and W. J. Teague, "Low-frequency resonant scattering of bubble clouds," Journal of Atmospheric and Oceanic Technology, vol. 17 , no. 6, pp. 847-853, 2000.

[39] K. W. Commander and A. Prosperetti, "Linear pressure waves in bubbly liquids: Comparison between theory and experiments," The Journal of the Acoustical Society of America, vol. 85, no. 2, pp. 732-746, 1989.

[40] C. Feuillade, "The attenuation and dispersion of sound in water containing multiply interacting air bubbles," The Journal of the Acoustical Society of America, vol. 99, no. 6, pp. 3412-3430, 1996.

[41] S. Espa, G. Caramanna, and V. Bouché, "Field study and laboratory experiments of bubble plumes in shallow seas as analogues of subseabed CO2 leakages," Applied Geochemistry, vol. 25, no. 5, pp. 696704, 2010.

[42] M. Römer, H. Sahling, T. Pape, G. Bohrmann, and V. Spieß, "Quantification of gas bubble emissions from submarine hydrocarbon seeps at the Makran continental margin (offshore Pakistan)," Journal of Geophysical Research: Oceans, vol. 117, no. C10, 2012.

[43] A. Nikolovska, H. Sahling, and G. Bohrmann, "Hydroacoustic methodology for detection, localization, and quantification of gas bubbles rising from the seafloor at gas seeps from the eastern Black Sea," Geochemistry, Geophysics, Geosystems, vol. 9, no. 10, 2008.

[44] C. L. Monjo, H. Nguyen, and H. A. Deferrari, "Modulations of detectable pulse response time spread in shallow water resulting from a combination of sound-speed variability and bottom loss," The Journal of the Acoustical Society of America, vol. 102, no. 4, pp. 2083-2097, 1997.

[45] E. C. Monahan and M. Lu, "Acoustically relevant bubble assemblages and their dependence on meteorological parameters," IEEE Journal of Oceanic Engineering, vol. 15, no. 4, pp. 340-349, 1990. 